\section{Zur Rolle von Akzeptanz und Akzeptabilität von Technik bei der Bewältigung von Technik- konflikten}

\author{
von Armin Grunwald, ITAS
}

Technischer Fortschritt führt neben den vielen erwünschten Folgen auch zu mancherlei Zumutungen, welche die Akzeptanz verringern. Technikakzeptanz kann verschieden konzeptualisiert werden: Zum einen kann gefragt werden, wie groß die Akzeptanz von Technik in der Gesellschaft ist und von welchen Faktoren sie abhängt; zum anderen kann thematisiert werden, unter welchen Bedingungen Technikakzeptanz normativ erwartet werden könne. Ist die erste Frage empirisch-sozialwissenschaftlich $\mathrm{zu}$ beantworten, verbirgt sich hinter der zweiten die Position normativer Ethik. Die in den 1990er Jahren zwischen Vertretern beider Positionen geführte heftige Debatte ist zu keinem abschließenden Ende gekommen. In diesem Beitrag werden die Streitpunkte erläutert und notwendige Differenzierungen vorgenommen, bevor ein Lösungsansatz skizziert wird.

\section{Ausgangssituation und Fragestellung}

Dass wissenschaftlicher Fortschritt und technische Innovationen zur Sicherung von Wohlstand und Wettbewerbsfähigkeit, aber auch für weitere Fortschritte in der Medizin und für eine nachhaltige Entwicklung erforderlich sind, ist weitgehend Konsens. Sie haben jedoch nicht ausschließlich positive Seiten. Nicht intendierte Nebenfolgen von Technik wie vor allem Risiken neuer Technologien für die natürliche Umwelt, für die menschliche Gesundheit oder für das kollektive Zusammenleben gehören zu den gesellschaftlichen Erfahrungen der letzten Jahrzehnte. Das Ozonloch, die katastrophale Geschichte der Asbestnutzung und die großen Unglücksfälle wie Bhopal oder Tschernobyl sind bekannte Beispiele (Harremoes et al. 2002 mit einer Reihe von einschlägigen Fallstudien). Dass Technikakzeptanz in den meisten Industrieländern zu einem Thema der öffentlichen Debatte geworden ist, liegt zu einem Teil - sicher nicht ausschließlich - an derartigen Erfahrungen. Sobald der technische Fortschritt zumindest partiell als riskant oder subjektiv nachteilig empfunden wird, sinkt - wenig überraschend die Zustimmung. Dann wird auch die gesellschaftliche Verteilung von Vorteilen und Nachteilen der betreffenden Technik zum kontroversen Thema, genauso wie die Differenz zwischen Entscheidern über Technik und den von den Entscheidungen Betroffenen, von denen Akzeptanz erwartet wird (vgl. die Einleitung zu diesem Schwerpunkt).

Auf der konzeptionellen Ebene gab es in den 1990er Jahren eine lebhafte Diskussion über Technikakzeptanz. Sie legte ihren Schwerpunkt auf die Akzeptanz von Technikrisiken und wurde von Philosophen einerseits und empirisch argumentierenden Sozialwissenschaftlern andererseits getragen. Während die Philosophen die Ethik als Ausgangspunkt wählten und einen normativen, von Rationalitätsstandards geprägten Umgang mit Technikrisiken favorisierten und hierfür das Konzept der Akzeptabilität entwickelten (Gethmann, Mittelstraß 1992; Gethmann, Sander 1999), setzten die Sozialwissenschaftler, die der Technikfolgenabschätzung (TA) nahe standen, auf die empirische Akzeptanz der Technikrisiken und argumentierten für eine sozialverträgliche Technikgestaltung (Alemann, Schatz 1986). Der vorliegende Beitrag schließt an diese Kontroverse an und versucht, durch eine veränderte „Rahmung“ des Problems zu einer Lösung zu gelangen.

\section{Akzeptanz oder Akzeptabilität von Technik?}

Die Erfahrung schwerwiegender Technikkonflikte, die in einigen Ländern punktuell bürgerkriegsähnliche Ausmaße erreichten, legt nahe zu fragen, ob man diese Konflikte nicht a prio$r i$ vermeiden, also bereits ihre Entstehung verhindern könnte statt später die Folgen dieser Konflikte teurer kurieren zu müssen: vorzubeugen statt im Ernstfall die Polizei rufen zu müssen. Auch angesichts des nach allen empirischen Ergebnissen geringen Vertrauens in die Fähigkeit des Staates, die technische Entwicklung erfolgreich zu regulieren (z. B. Hennen 1997; Renn, Zwick 1997), erscheint es prima facie als sinnvoll, die Risiken auf ein sowieso bereits akzeptiertes Maß zu begrenzen. Staatliche Regulierung wäre in diesem Fall gar nicht erforderlich. 
Die Idee akzeptanzorientierter Technikgestaltung bestand darin, die angenommene Technikakzeptanz bereits in der Technikentwicklung zu berücksichtigen. Durch prospektive Untersuchungen sei herauszufinden, welche Technik (einschließlich ihrer Risiken und sonstiger Nachteile) faktisch wohl akzeptiert würde. Daraus resultierte die Aufgabe für Ingenieure, Technik innerhalb des dadurch vorgegebenen Akzeptanzrahmens zu entwickeln: „Insofern müssen die Ergebnisse von Einstellungs- und Akzeptanzforschung zweifelsohne ein nicht unbedeutender Teil von Sozialverträglichkeitsprüfungen sein; eine Rückbindung zum Meinungsklima ist unabdingbar" (Jaufmann 1999, S. 220). Erfahrungen mit der mangelnden Prognostizier- oder Extrapolierbarkeit des Akzeptanzverhaltens sowie Probleme der Interpretation des Akzeptanzverhaltens als einer komplexen Größe (Gloede 1987) führten jedoch zu einer prozeduralen Wende (Simonis 1999). Danach geht es nicht mehr um eine prospektive „Messung“ und Extrapolation des Akzeptanzverhaltens, sondern darum, die von der Technikentwicklung Betroffenen (Konsumenten, Bürger, politische Parteien, Behörden, Verbände, soziale Bewegungen etc.) in die Entscheidungsprozesse einzubeziehen. Die kategoriale Differenz zwischen Entscheidern und von der Entscheidung Betroffenen sollte auf diese Weise zumindest teilweise aufgehoben werden.

Gegen das akzeptanzorientierte Konzept wurde seitens der philosophischen Ethik eingewandt, dass einerseits faktische Technikakzeptanz nichts über die ethische Rechtfertigbarkeit der Technik aussage, und dass andererseits Nichtakzeptanz nicht notwendigerweise ein Grund sei, eine spezifische Technik trotz nicht vorhandener Akzeptanz zu implementieren. Vom Faktischen auf das Gesollte zu schließen, sei ein naturalistischer Fehlschluss (Gethmann, Sander 1999). Deswegen sei in Technikkonflikten nicht das Konzept der faktischen Akzeptanz, sondern dass der normativen Akzeptabilität grundlegend: „Akzeptabilität ist ein normativer Begriff, der die Akzeptanz von risikobehafteten Optionen mittels rationaler Kriterien des Handelns unter Risikobedingungen festlegt" (dies. 1999, S. 146). Auf diese Weise werden die Zumutbarkeit von Nebenfolgen technischer Entwicklungen wie Lärm oder stoffliche Emissionen, die Kodifizierung von solchen „Zumutbarkeiten“ durch Grenzwerte wie Umwelt- oder Sicherheitsstandards, aber auch die Bedingungen dieser ,normativen“ Akzeptanz in den konzeptionellen Mittelpunkt der Betrachtung gestellt.

Das Akzeptabilitätskonzept arbeitet mit Rationalitätsstandards, die als normative Erwartungen an handelnde und urteilende Personen gerichtet werden. Konsistenzfragen im Umgang mit verschiedenen Risikotypen spielen eine wesentliche Rolle (Gethmann, Mittelstraß 1992), Inkonsistenz gilt als Indikator mangelnder Rationalität. Neue (z. B. technikbedingte) Risiken werden durch Konsistenzüberlegungen in Beziehung zu bereits - z. B. in der Lebenswelt - etablierten Risiken gesetzt und vergleichbar gemacht. Das resultierende Prinzip der pragmatischen Konsistenz lautet: „Hat jemand durch die Wahl einer Lebensform den Grad eines Risikos akzeptiert, so darf dieser auch für eine zur Debatte stehende Handlung unterstellt werden“ (Gethmann, Sander 1999, S. 146 f.).

Die Konsequenzen dieser Kontroverse sind weit reichend. Je nachdem, ob gefragt wird „Wird bestimmte Technik akzeptiert werden?"“ oder ,Soll bestimmte Technik akzeptiert werden?" wird die gesellschaftliche Diskussion über Technik und ihre Risiken jeweils in einen deutlich anderen Rahmen gestellt. Im Folgenden wird die These vertreten, dass beide Ansätze zwar auf zutreffende Sachverhalte aufmerksam machen, in ihrer jeweiligen Beschränktheit jedoch keine adäquate Konzeptualisierung der Risikothematik darstellen.

\section{Kritik}

Akzeptanzforschung zu Technikrisiken gibt wesentliche Einsichten in empirische Verhaltensweisen und in „Befindlichkeiten“ in der Bevölkerung, ihre zeitliche Entwicklung und ihre wesentlichen Einflussfaktoren. Damit stellt sie wichtige Informationen für politische Handlungsweisen bereit. Die Akzeptanzorientierung kann aber das grundlegende Problem des demokratischen Umgangs mit Technikkonflikten nicht lösen (vgl. Grunwald 2000a, b), es stellen sich folgende Probleme:

1. Extrapolationsproblem: Es kann immer nur die jeweils gegenwärtige Akzeptanzsituation empirisch erfasst und in der betreffenden 
technikrelevanten Entscheidung berücksichtigt werden (auch das ist methodisch schon schwierig genug). Weil Technikakzeptanz zeitlich stark schwanken kann, schließt die Berücksichtigung des gegenwärtigen Akzeptanzverhaltens zukünftige Technikkonflikte nicht aus.

2. Stabilitätsproblem: Die zeitliche Instabilität der Technikakzeptanz (Jungermann, Slovic 1993) hätte für ein akzeptanzorientiertes Vorgehen starke Diskontinuitäten zur Folge und könnte Planungssicherheit weder für Investoren noch für Technikkonsumenten geben (Grunwald 2000b).

3. Aggregationsproblem: Das Arrow-Theorem aus der Entscheidungstheorie (Arrow 1963) zeigt, dass unter sehr allgemeinen Voraussetzungen individuelle Präferenzen nicht widerspruchsfrei zu einer wohl definierten Gesamtnutzenfunktion aggregiert werden können. Übertragen auf unser Problem heißt dies, dass kaum Aussicht besteht, die individuellen Präferenzen hinsichtlich der Technikakzeptanz in einer pluralistischen, von Wertekonflikten durchzogenen Gesellschaft $\mathrm{zu}$ einem konsistenten Gesamtbild zusammenzufügen. Entweder müssen substantielle Wertkonsense vorausgesetzt werden (wofür es keine Hinweise gibt), oder es können nicht alle Präferenzen gleichermaßen befriedigt werden. Wäre letzteres der Fall, dann stünde die Gesellschaft wieder vor den Technikkonflikten, die gerade vermieden werden sollten.

Auf der anderen Seite führt auch die konsequente Durchführung eines rationalitätstheoretischen Akzeptabilitätsansatzes zu ganz erheblichen Problemen - auch wenn sie von der zutreffenden Beobachtung ausgeht, dass das Gelingen von Kommunikation und Kooperation unzweifelhaft die Einhaltung bestimmter Rationalitätsstandards einschließlich eines gewissen Maßes an Konsistenz erfordert.

1. Skalenproblem: Der Akzeptabilitätsansatz basiert auf Vergleichen von Risiken auf einer einheitlichen Skala. Damit unterstellt er einen „objektiven“ Risikobegriff (Gethmann, Sander 1999). Die Projektion verschiedener Risiken auf eine einheitliche Skala ist jedoch kein wertneutraler Vorgang. Bereits die Quantifizierung von Schadensgrößen und Eintrittswahrscheinlichkeiten stößt häufig auf ethische und politische Fragen (Grunwald 1994) und ist daher selbst Gegenstand von gesellschaftlichen Kontroversen und Konflikten. Dies gilt a fortiori für die Frage, welche Risiken in welchen Situationen zu welchen Zwecken als abwäg- und vergleichbar angesehen werden und welche nicht.

2. Kontextualisierungsdefizit: Bei einem Akzeptabilitätsansatz, der auf dem „objektiven“ Risikobegriff basiert, werden Risiken letztlich auf die Form von numerischen Größen gebracht und so dekontextualisiert. Es gerät aus dem Blick, dass Risiken immer Risiken für jemanden sind. Insbesondere verschwindet in dieser Dekontextualisierung die Differenz zwischen Entscheidern und Betroffenen.

3. Mangelnde Operationalisierbarkeit: Die unmittelbare Heranziehung des Prinzips der pragmatischen Vernunft zur Regelung allgemein verbindlicher Angelegenheiten wäre nur möglich, wenn entsprechende Konsistenzforderungen im demokratischen Regelwerk akzeptiert und kodifiziert wären. Das ist aber nicht der Fall. Im demokratischen System werden die Staatsbürger in der Wahrnehmung ihrer staatsbürgerlichen Rechte gegenwärtig jedoch keineswegs an Rationalitätsstandards gemessen. Niemand muss vor einem Wahlgang oder in der Teilnahme an der öffentlichen Meinungsbildung einen Rationalitätstest bestehen. Inkonsistenzen in der Lebensführung sind kein Hemmnis zur Ausübung staatsbürgerlicher Rechte) - auch wenn offensichtliche Inkonsistenzen die Glaubwürdigkeit in Argumentationssituationen unzweifelhaft herabsetzen.

\section{Lösungsvorschlag}

Auch wenn keiner der beiden Ansätze zur Bewältigung gesellschaftlicher Technikkonflikte als hinreichend einzustufen ist, können an dieser Stelle folgende Punkte festgehalten werden:

- Allgemein verbindliche, demokratisch legitimierte Entscheidungen über technische Risiken müssen letztlich akzeptiert werden.

- Der Durchsetzungsmacht des Staates mit hoheitlichen, letztlich polizeilichen Mitteln sind Grenzen gesetzt, sowohl hinsichtlich der Kosten als auch hinsichtlich der Glaubwürdigkeit. 
- Eine Ausrichtung der Entscheidungsabläufe auf die mutmaßliche Technikakzeptanz löst das Problem nicht.

Zur Vorbereitung der vorgeschlagenen Lösung (s. Kap. 4.2) wird im Folgenden zunächst der Kern des Problems der Technikakzeptanz näher bestimmt.

\subsection{Differenzierung}

Zunächst ist festzuhalten, dass Technikakzeptanz ein für viele Zwecke zu pauschaler Begriff ist. Vielmehr sind verschiedene Situationen zu unterscheiden, in denen mit der Akzeptanzproblematik konzeptionell jeweils unterschiedlich umgegangen werden muss. Prämisse ist im Folgenden, dass in der Problematisierung von „Akzeptanz“ immer ein „trotzdem“ mitschwingt: Sowohl bei erwarteter wie auch bei faktischer Technikakzeptanz ist immer zu berücksichtigen, dass diese trotz bestimmter Risiken oder anderer Nachteile erwartet bzw. erzielt wurde. Ohne diese „dunkle Seite“ des technischen Fortschritts und entsprechende Zumutungen bestünde kein Bedarf, über Technikakzeptanz nachzudenken. Aus dieser semantischen Einsicht folgt sofort, dass auch die Verteilung von Vor- und Nachteilen der jeweiligen technischen Entwicklung zum Thema wird. Die daran anschließende Frage lautet: Um welche Vor- und Nachteile für wen geht es? An dieser Stelle sei die folgende (sicher nicht erschöpfende) Abschichtung vorgeschlagen:

- Zumutungen, die individuell kontrolliert werden können (wie Motorradfahren, Risikosportarten oder vielleicht zukünftig eine Urlaubsreise in den Weltraum): Hier hält der Betroffene selbst die Entscheidung darüber in den Händen, ob und welche Risiken oder Nachteile er hinzunehmen bereit ist. Über die individuelle Ebene hinaus gehende Aspekte gewinnen erst dann an Bedeutung, wenn die Übernahme der Kosten im Schadensfalle zu klären ist. So stellt sich z. B. regelmäßig wiederkehrend die Frage, ob Krankenkassen Verletzungen erstatten sollen, die bei der Ausübung von Risikosportarten entstehen.

- Zumutungen mit einfachen Ausweichmöglichkeiten: Bestimmte Zumutungen können relativ einfach umgangen werden. Hierzu würden mögliche gesundheitliche Risiken gentechnisch veränderter Nahrungsmittel gehören. Die Kennzeichnungspflicht eröffnet dem Kunden die Möglichkeit, auf nicht gentechnisch veränderte Produkte auszuweichen. Voraussetzung ist dabei, dass genügend Alternativprodukte vorhanden sind und der Einzelne auf die Korrektheit der Kennzeichnung vertraut. Erst unter diesen Voraussetzungen wird niemandem alternativlos etwas zugemutet.

- Zumutungen mit beschwerlichen Ausweichmöglichkeiten: Manchen Zumutungen kann man zwar ,prinzipiell“, aber faktisch oft nicht ausweichen. Betroffene werden stattdessen ,exponiert“. Klassisch sind hier Standortprobleme von Müllverbrennungsanlagen, radioaktiven Endlagern, Chemiefabriken oder in früheren Zeiten auch von Kernkraftwerken. Prinzipiell besteht hier zwar eine Ausweichmöglichkeit, man kann ja wegziehen, aber in der Regel doch nur mit erheblichen sozialen und ökonomischen Belastungen.

- Zumutungen ohne Ausweichmöglichkeit: Einige Technikfolgen sind diffus verteilt. Dazu gehören z. B. das Ozonloch, die schleichende Grundwasserverschmutzung, die Degradierung von Böden, die Akkumulation von Schadstoffen in der Nahrungsmittelkette sowie Lärm und Feinststaubbelastung. In diesen Fällen hilft weder eine Kennzeichnung noch die Verlagerung des Standortes. Diesen Zumutungen kann man nicht entgehen. Allerdings kann es Ungleichheiten der Exposition geben (z. B. durch bevorzugte und benachteiligte Wohnsituationen), die ein Expositionsgefälle schaffen.

Die Möglichkeit der Einflussnahme auf die Exposition gegenüber den Zumutungen des technischen Fortschritts bestimmt stark den Charakter der entsprechenden Situationen. In marktnahen Situationen ist der Einfluss der Betroffenen auf Akzeptanz oder Ablehnung der Zumutungen (z. B. durch die Wahl zwischen Alternativen im Supermarktregal) groß. Anders sieht dies jedoch aus, sobald die Ausweichmöglichkeiten gegenüber Zumutungen erschwert werden oder ganz verschwinden, sobald also die Exposition nicht mehr oder nur noch unter größeren persönlichen Opfern durch die Betroffenen beeinflusst werden kann. Dies betrifft einerseits Standortfragen. Anwohner von Che- 
miefabriken oder von Militäranlagen wollen mit Recht nicht durch die Nachbarschaft gefährdet werden oder Nachteile für ihre Lebensqualität in Kauf nehmen. Hier wäre es ziemlich absurd, auf die freiwillige faktische Akzeptanz der entsprechenden Zumutungen bei den Betroffenen zu setzen. Warum sollte jemand freiwillig eine Müllverbrennungsanlage vor seiner Haustür akzeptieren? Das wäre vom individuellen Standpunkt aus zumeist höchst irrational. Aber irgendein Standort muss, wenn z. B. eine Müllverbrennungsanlage gebaut werden soll, doch letztlich faktisch akzeptiert werden. Dies wiederum bedeutet, dass Betroffene etwas von anderen Entschiedenes bereit sein müssen zu akzeptieren. Hier zeigt sich der Kern des Problems als Legitimationsproblem (Grunwald 2000a): Warum soll gerade eine Personengruppe am Standort A und nicht eine andere Personengruppe die befürchteten Zumutungen tragen (z. B. bei Standortfragen eines radioaktiven Endlagers; vgl. dazu Hocke, Grunwald 2005)?

Verschärft stellt sich dieses Problem im Falle ,diffus verteilter" Zumutungen oder Risiken. Warum sollte jemand unter individuellen Präferenzen eine Feinststaubbelastung akzeptieren, deren medizinische Auswirkungen unklar, aber jedenfalls nicht positiv sein werden? Wenn die Betroffenen identisch mit den Nutznießern wären, könnte man noch subjektive Risiko/Nutzen-Erwägungen durchführen. Aber diese Prämisse ist in der Regel nicht erfüllt. Vielmehr müssen in solchen Fällen gesellschaftsweit verbindliche Standards (wie Risikogrenzen, Sicherheitsstandards, Umweltstandards etc.) gesetzt werden. Gesellschaftsweit, und nicht vom Standpunkt individueller Akzeptanz- oder Nichtakzeptanzhaltungen muss über die Grenzen der Belastbarkeit und über Abwägungen zwischen Risiken und Chancen verbindlich befunden werden.

\subsection{Legitimation und Akzeptanz}

Es geht daher im „harten“ Kern der Debatte zu Technikakzeptanz einerseits um die unfreiwillig einzugehenden Zumutungen und ihre gesellschaftliche Verteilung, die der - im Prinzip nicht in Frage gestellte - technische Fortschritt mit sich bringt, sowie andererseits um die gesellschaftsweit verbindliche Regelung dieser Zumutungen und Zumutbarkeiten. Das bedeu- tet, dass wir es hier mit einem Problem zu tun haben, das auf der Ebene gelöst werden muss, auf der wir kollektive Verbindlichkeiten regeln: auf der Ebene demokratischer Meinungsbildung und Entscheidungsfindung und damit im politischen Teilsystem mit seinen spezifischen Stärken und Schwächen.

Damit ist die Frage verschoben hin zu der Aufgabe, legitime und verbindliche Entscheidungen über Technik zu treffen in der Erwartung, dass diese akzeptiert werden. Hier stellt sich also die Herausforderung der Akzeptabilität: Es geht letztlich um Zumutungen - allerdings, und das macht die hier vorgeschlagene Verschiebung der Perspektive deutlich, nicht mehr um eine Akzeptabilität unter Rationalitätsstandards, die an ein individuelles Handeln angelegt werden, sondern um eine Akzeptabilität, die ihre Basis in demokratisch legitimierten Entscheidungen hat, also eine demokratietheoretische und keine rationalitätstheoretische Akzeptabilität. Gemeinsam ist beiden die Tatsache, dass es sich um Akzeptanzzumutungen handelt; grundverschieden ist jedoch die argumentative Basis, auf der sie aufruhen.

Die Legitimation von verbindlichen politischen Entscheidungen über Technik einschließlich ihrer Zumutungen hängt normativ mit ihrer Akzeptanz zusammen: Legitime Entscheidungsresultate beanspruchen akzeptiert $\mathrm{zu}$ werden; ihre Akzeptanz darf also mit einiger Berechtigung erwartet werden. So sind z. B. bei der Planung einer Autobahn akzeptierte und legitimierte Verfahren (z. B. Planfeststellungsverfahren) zu beachten mit der Konsequenz, dass die resultierende Entscheidung auch von den Betroffenen akzeptiert werden soll, wenn das Verfahren korrekt durchgeführt wurde. Mit Hilfe solcher Verfahren definiert die Gesellschaft, bis zu welchem Maß die Akzeptanz bestimmter Zumutungen unter „öffentlichem Interesse“ erwartet werden kann. Dies reicht bis hin zu der Situation, dass bestimmten Personen oder Gruppen die Lasten solcher Entscheidungen aufgebürdet werden können. Diese Verfahren klären auch, was „Verlierern“" zugemutet werden darf und ob Kompensationen vorgesehen sind.

Der Kern von Demokratie als Entscheidungssystem besteht gerade nicht darin, dass jede demokratisch legitime Entscheidung auf vollständige Akzeptanz in dem Sinne stoßen muss, dass sie verträglich mit den Präferenzen 
und Interessen aller Betroffenen sein soll. Demokratische Entscheidungen müssen Probleme mit Gewinnern und Verlierern und einer entsprechenden Zumutungsproblematik bewältigen können. Entscheidend ist - und dies ist Teil des demokratischen Selbstverständnisses (Luhmann 1983, S. 29) -, dass Resultate legitimierter Entscheidungsprozesse auch dann akzeptiert werden ,sollen“, wenn sie unter den individuellen Präferenzen unwillkommen sind. Die Akzeptanz der Prozeduren - und hier ist Akzeptanz tatsächlich entscheidend - führt somit zur Legitimation der Resultate dieser Prozeduren und somit zur berechtigten Erwartung, dass sie akzeptiert werden.

„[Es] gehört die Fraglosigkeit legitimer Geltung bindender Entscheidungen zu den typischen Kennzeichen des modernen politischen Systems als eine Art von Grundkonsens, der ohne Übereinstimmung über das im Einzelfall sachlich Richtige erreicht werden kann und das System stabilisiert" (Luhmann 1983, S. 29 f).

Übertragen auf Zumutungen durch den technischen Fortschritt (z. B. technische Risiken) bedeutet dies eine Verlagerung der Akzeptanzanforderungen von den „Umfragen“ über mutmaßliche Technikakzeptanz auf die Ebene der demokratisch legitimierten und prozeduralen Bewältigung der unvermeidlichen Zumutungen der Technik. Ein solches System, in dem durch akzeptierte Verfahren normativ akzeptable und dann auch faktisch akzeptierte Entscheidungsresultate erzeugt werden, stößt jedoch gelegentlich an Grenzen. Dies ist vor allem dann zu beobachten, wenn die normativ erwartete Akzeptanz faktisch massiv ausbleibt (wie im Fall der bekannten Technikkonflikte um Kernenergie, Gentechnik und radioaktive Endlager). Dann sind die Prozeduren selbst in einem gesellschaftlichen Lernprozess zu ändern. Dieser Lernprozess sollte als Resultat neue oder modifizierte, wiederum akzeptierte Prozeduren als neue Basis legitimierter Entscheidungen hervorbringen. Die endlosen und bislang fruchtlosen Diskussionen um eine Endlagerung radioaktiver Abfälle in vielen Ländern sind ein ausgezeichnetes Beispiel dafür, dass auch formal legitimierte Beschlüsse derart massiv auf Ablehnung stoßen können, dass ihre Umsetzung verhindert wird. In diesem Fall bedarf es weiterentwickelter Prozeduren (Hocke, Grunwald 2005).

\subsection{Konsequenzen für die Technikfolgen- abschätzung}

Technikfolgenabschätzung (TA) ist auf Wissen über Technik- und Risikoakzeptanz angewiesen. Bereits allgemeine Zielsetzungen der TA (wie die präventive Vermeidung oder Verringerung von Technikrisiken $\mathrm{zu}$ ermöglichen, Technikkonflikte zu deeskalieren oder zu lösen) bedürfen des Wissens um Bedingungen von Technikakzeptanz in der Gesellschaft. Es gibt jedoch nach dem Gesagten keinen direkten Weg von der faktischen Technikakzeptanz zum gesellschaftlichen Umgang mit neuen technischen Risiken, die der Gesellschaft bzw. bestimmten Gruppen zugemutet werden und insofern technischer Fortschritt und Innovation als solche nicht infrage gestellt werden - auch zugemutet werden müssen.

Auf der anderen Seite bedarf die TA in der Bewertung von Technikrisiken des Rates der Ethik, um nicht in den naturalistischen Fehlschluss von faktischer Akzeptanz auf Akzeptabilität abzugleiten und simpel die normative Kraft des Faktischen anzuerkennen. Bis hinein in Regulierungsfragen spielen normative Aspekte eine Rolle - z. B. hinsichtlich der Anwendbarkeit und der Konsequenzen des Vorsorgeprinzips oder in der Frage, welche RisikoRisiko-Vergleiche mit welchen Begründungen zugelassen werden. Aber auch hier gilt, dass ethische Prinzipien (wie z. B. das Prinzip der pragmatischen Konsistenz) nicht unmittelbar als Anleitung zum Umgang mit Zumutungen des technischen Fortschritts herangezogen werden können. Die angedeutete demokratietheoretische Wendung des Problems führt zu folgenden Konsequenzen für die Behandlung von Technikrisiken in der TA:

1. Es ist zu differenzieren nach Zumutungsklassen. Der Grad, in dem Betroffene dabei ihre eigene Exposition gegenüber den $\mathrm{Zu}-$ mutungen und Risiken beeinflussen können, spielt eine erhebliche Rolle.

2. Die in der TA vollzogene, ,prozedurale Wende" in der Behandlung von Risikoakzeptanzproblemen entspricht der Diagnose, dass die gelingende Bestimmung von legitimierten Risikostandards eine Frage geeigneter Entscheidungsprozeduren ist.

3. In der TA zu solchen Fragen geht es also um die Beratung der Entscheidungsverfah- 
ren. Beratung meint hier sowohl Beratung in sachlicher, aber auch Beratung in normativer Hinsicht (Orientierungswissen).

4. Eine besondere Rolle kann dabei die Frage spielen, ob die etablierten Verfahren ausreichend sind oder nicht. In Fällen, in denen trotz legitimierter Entscheidungsverfahren die Resultate hartnäckig abgelehnt werden (dies ist praktisch weltweit der Fall in Standortsuchen radioaktiver Endlager, Hocke, Grunwald 2005), müssen neue Verfahren entwickelt werden.

\section{Literatur}

Alemann, U.; Schatz, H., 1986: Mensch und Technik. Grundlage und Perspektiven einer sozialverträglichen Technikgestaltung. Opladen: Westdeutscher Verlag Arrow, K., 1963: Social Choice and Individual Values. London: Yale University Press

Gethmann, C. F.; Mittelstraß, J., 1992: Umweltstandards. In: GAIA 1(1992), S. 16-25

Gethmann, C.F.; Sander, T., 1999: Rechtfertigungsdiskurse. In: Grunwald, A.; Saupe, S. (Hrsg.): Ethik in der Technikgestaltung. Praktische Relevanz und Legitimation. Heidelberg: Springer, S. 117-151

Gloede, F., 1987: Vom Technikfeind zum gespaltenen Ich. Thesen zur Technikakzeptanz. In: Lompe, K. (Hrsg.): Techniktheorie, Technikforschung, Technikgestaltung. Opladen: Westdeutscher Verlag, S. 233-261

Grunwald, A., 1994: Wissenschaftstheoretische Anmerkungen zur Technikfolgenabschätzung: Prognose- und Quantifizierungsproblematik. In: Journal of the General Philosophy of Science 25/1 (1994), S. 51-70

Grunwald, A., 2000a: Technik für die Gesellschaft von morgen. Möglichkeiten und Grenzen gesellschaftlicher Technikgestaltung. Frankfurt a. M.: Campus

Grunwald, A., 2000b: Technology Policy Between Long-Term Planning Requirements and ShortRanged Acceptance Problems. In: Grin, J.; Grunwald, A. (Hrsg.): Vision assessment: shaping technology in $21^{\text {st }}$ century society. Heidelberg: Springer, S. $99-148$

Harremoes, P.; Gee, D.; MacGarvin, M.; Stirling, A.; Keys, J.; Wynne, B.; Guedes Vaz, S. (Hrsg.), 2002: The Precautionary Principle in the $20^{\text {th }}$ century. Late Lessons from early warnings. London: Earthscan Publications
Hennen, L., 1997: Technikakzeptanz und Kontroversen über Technik. Ambivalenz und Widersprüche: Die Einstellung der deutschen Bevölkerung zur Technik. TAB-Arbeitsbericht Nr. 54, Berlin

Hocke, P., Grunwald, A. (Hrsg.), 2005: Wohin mit dem radioaktiven Abfall? Berlin: edition sigma

Jaufmann, D., 1999: Technikakzeptanzforschung. In: Bröchler, S.; Simonis, G.; Sundermann, K. (Hrsg.): Handbuch Technikfolgenabschätzung. Berlin: edition sigma, S. 205-226

Jungermann, H.; Slovic, P., 1993: Charakteristika individueller Risikowahrnehmung. In: Krohn, W.; Krücken, G. (Hrsg.): Riskante Technologien: Reflexion und Regulation. Frankfurt a. M.: Campus, S. $79-100$

Luhmann, N., 1983: Legitimation durch Verfahren. Frankfurt: Suhrkamp

Renn, O.; Zwick, M., 1997: Risiko- und Technikakzeptanz. Berlin: Springer

Simonis, G., 1999: Sozialverträglichkeit. In: Bröchler, S.; Simonis, G.; Sundermann, K. (Hrsg.): Handbuch Technikfolgenabschätzung. Berlin: edition sigma, S. 105-118

\section{Kontakt}

Prof. Dr. Armin Grunwald

Forschungszentrum Karlsruhe $\mathrm{GmbH}$

Institut für Technikfolgenabschätzung und Systemanalyse (ITAS)

Postfach 3640, 76021 Karlsruhe

Tel.: +49 (0) 7247 / 82 - 2500

Fax: +49 (0) 7247 / 82 - 4806

E-Mail: grunwald@itas.fzk.de

Internet: http://www.itas.fzk.de 